\title{
miR-3666 suppresses cellular proliferation and invasion in colorectal cancer by targeting SATB2
}

\author{
DAQING YANG, RIZENG LI, JIANFU XIA, WENCAI LI and HONG ZHOU
}

Department of Colorectal Surgery, Wenzhou Central Hospital, Wenzhou, Zhejiang 325000, P.R. China

Received February 15, 2018; Accepted July 27, 2018

DOI: $10.3892 / \mathrm{mmr} .2018 .9540$

\begin{abstract}
MicroRNA-3666 (miR-3666) acts as a tumor suppressor in cervical cancer, non-small cell lung cancer and thyroid carcinoma; however, the function of miR-3666 in colorectal cancer (CRC) remains largely unknown. In the present study, was demonstrated that miR-3666 was significantly downregulated in CRC tissues compared with in adjacent normal tissues by reverse transcription-quantitative polymerase chain reaction. Additionally, miR-3666 may serve as a prognostic biomarker for patients with CRC. Via functional experiments, the present study reported that miR-3666 overexpression significantly inhibited the proliferation, migration and invasion of CRC cells as determined by Cell Counting Kit- 8 and Transwell assays, and vice versa. In addition, miR-3666 was reported to directly target special AT-rich sequence binding protein 2 (SATB2) in CRC cells; overexpression of miR-3666 significantly suppressed the expression of SATB2 in CRC cells as determined by western blotting. Furthermore, an inverse correlation was observed between the expression levels of miR-3666 and SATB2 in CRC tissues. Restoration of SATB1 expression significantly reversed the effects of miR-3666 mimic on CRC cells. In summary, the results of the present study indicated that miR-3666 may serve as a tumor suppressor in CRC by targeting SATB2.
\end{abstract}

\section{Introduction}

As the third most common malignancy worldwide, colorectal cancer (CRC) leads to numerous cases of cancer-associated mortalities annually (1). Several factors, including genetic mutations, inflammation, alcohol consumption and smoking may induce the development of CRC (2). Therapeutic strategies have been developed; however, the 5-year survival rate of patients with CRC remains unsatisfactory (3). Tumor

Correspondence to: Dr Hong Zhou, Department of Colorectal Surgery, Wenzhou Central Hospital, 32 Dajian Lane, Lucheng, Wenzhou, Zhejiang 325000, P.R. China

E-mail: hongzhou0101@163.com

Key words: miR-3666, special AT-rich sequence binding protein 2, colorectal cancer, proliferation, invasion metastasis and recurrence are the main factors that affect the treatment of patients with CRC (4). Numerous signaling pathways and molecules have been reported to be associated with the development or progression of CRC, including the Notch and adenomatous polyposis coli tumor suppressor signaling pathways $(5,6)$; however, the molecular mechanisms underlying the carcinogenesis of CRC remain largely unknown. Furthermore, it is necessary to determine effective biomarkers and therapeutic targets for CRC intervention.

MicroRNAs (miRNAs/miRs) are a collection of short noncoding RNAs that regulate gene expression by targeting the 3'-untranslated region (UTR) of target mRNAs via base-pairing (7). The functions of miRNAs have been widely reported, such as their involvement in various physiological and pathological processes (8), including the cell-cycle, proliferation, apoptosis and metastasis $(9,10)$. The dysregulation of miRNAs has been demonstrated to be associated with the carcinogenesis of human cancers, including CRC (11). Increasing evidence has demonstrated that miRNAs are aberrantly expressed in CRC and regulate cancer progression (12-15). Some miRNAs have been reported as potential biomarkers or targets for the diagnosis, prognosis and therapy of CRC (16). Thus, understanding the function and mechanism of miRNAs in CRC may aid the development of effective therapeutic methods for the treatment of CRC.

Previous studies have demonstrated that miR-3666 was detected in non-small cell lung cancer, thyroid carcinoma and cervical cancer (17-19). Whether miR-3666 regulates the progression of CRC remains unknown. In the present study, it was reported that miR-3666 was significantly downregulated in CRC tissues and may have suppressed the proliferation, migration and invasion of CRC cells by targeting special AT-rich sequence binding protein 2 (SATB2). The findings of the present study suggested that miR-3666 may be a promising therapeutic target for the treatment of CRC.

\section{Materials and methods}

Patient samples. A total of 53 CRC and 53 adjacent non-cancerous specimens (28 males and 25 females; aged 33-67 years old with a median age of 54 years) were obtained from patients undergoing surgical resection at the Wenzhou Central Hospital (Wenzhou, China) between January 2014 and December 2016. Patients who had previously been subjected to chemotherapy and radiotherapy were excluded from the present study. miR 
expression was categorized into low and high groups according to the mean value, which were then analyzed by Kaplan Meier analysis. The dissected tissue specimens were immediately frozen in liquid nitrogen and stored at $-80^{\circ} \mathrm{C}$. All participants provided written informed consent for the collection of tissue samples. The present study was reviewed and approved by the Institutional Human Experiment and Ethics Committee of Wenzhou Central Hospital. The experimental procedures were performed in accordance with the Declaration of Helsinki.

Cell lines and cell culture. The human CRC cell lines, HT29, HCT116, SW480 and SW620 were obtained from the Cell Bank of Type Culture Collection of the Chinese Academy of Sciences (Shanghai, China). Human normal colon mucosal epithelial cells (NCM460) were obtained from Incell Corporation LLC (San Antonio, TX, USA). The cells were cultured in Dulbecco's modified Eagle's medium (DMEM; GE Healthcare Sciences, Logan, UT, USA), supplemented with $10 \%$ fetal bovine serum (FBS, Invitrogen; Thermo Fisher Scientific, Inc., Waltham, MA, USA) and $1 \%$ penicillin/streptomycin (Thermo Fisher Scientific, Inc.) in a humidified incubator at $37^{\circ} \mathrm{C}$ containing 5\% CO 2 . HCT116 and SW480 cells were selected for subsequent functional investigation, as these cells expressed the lowest miR-3666 expression levels.

Cell transfection. miR-3666 mimics (5'-CAGUGCAAG UGUAGAUGCCGA-3'), inhibitors (5'-UCGGCAUCUACA CUUGCACUG-3') and negative control (miR-NC; 5'-UCA CAACCUCCUAGAAAGAGUAGA-3') were obtained from Shanghai GenePharma Co., Ltd. (Shanghai, China). A pcDNA3.1-SATB2 overexpressing plasmid (Shanghai GenePharma Co., Ltd.) was synthesized to overexpress SATB2 by cloning the coding sequence into pcDNA3.1 vector as previously reported (20); empty pcDNA3.1 vectors served as a negative control. Transfection was performed using Lipofectamine ${ }^{\circledR} 2000$ (Invitrogen; Thermo Fisher Scientific, Inc.) according to the manufacturer's protocol. To restore SATB2 expression, miR-3666 mimics (100 nM) and either SATB2-overexpressing plasmids $(1 \mu \mathrm{g})$ or empty vectors $(1 \mu \mathrm{g})$ were co-transfected into $2 \times 10^{6} \mathrm{CRC}$ cells. A total of $48 \mathrm{~h}$ post-transfection, the efficiency was confirmed using RT-qPCR according to the aforementioned protocol.

Cell proliferation. For the Cell Counting Kit-8 (CCK-8) assay, cells were seeded into a 96 -well plate at a density of $1 \times 10^{4}$ cells/well and cultured for $48 \mathrm{~h}$ at $37^{\circ} \mathrm{C}$ containing $5 \%$ $\mathrm{CO}_{2}$ in complete DMEM; cells were transfected as aforementioned with miR-3666 mimics, inhibitor or miR-NC for $48 \mathrm{~h}$. Then, $10 \mu \mathrm{l}$ CCK-8 solution (Sigma-Aldrich; Merck KGaA, Darmstadt, Germany) was added to each well. Following incubation for $1 \mathrm{~h}$ at $37^{\circ} \mathrm{C}$, the optical density at $450 \mathrm{~nm}$ was detected using an enzyme-linked immunoassay analyzer (BioTek Instruments, Inc. Winooski, VT, USA).

Bioinformatics analysis. Putative target genes of miR-3666 were predicted using TargetScan 7.0 tool (http://www. targetscan.org/vert_70/).

Transwell assay. A Transwell chamber with $8-\mu \mathrm{m}$ pore size (Corning Incorporated, Corning, NY, USA) was used for the analysis of cell migration and invasion. Following transfection as aforementioned with miR-3666 mimics, inhibitor or miR-NC for $36 \mathrm{~h}$, CRC cells $\left(5 \times 10^{4}\right.$ cells) suspended in serum-free DMEM were plated into the upper chamber with (for invasion analysis) or without (for migration analysis) Matrigel. DMEM medium and $10 \%$ FBS was added to the lower chamber as a chemoattractant. Cells were incubated at $37^{\circ} \mathrm{C}$ for $24 \mathrm{~h}$, and the remaining cells on the upper chambers were removed with cotton swabs. The invaded or migrated cells on the lower chamber surface were fixed with $100 \%$ methanol for $30 \mathrm{~min}$ at $25^{\circ} \mathrm{C}$ and stained with $0.5 \%$ crystal violet for $30 \mathrm{~min}$ at $25^{\circ} \mathrm{C}$. The number of invaded and migrated cells were observed and counted in 5 randomly selected fields from each chamber under an inverted microscope (magnification, x100; Olympus Corporation, Tokyo, Japan).

Reverse transcription-quantitative polymerase chain reaction (RT-qPCR). Total RNA from CRC tissues and cultured cell lines was extracted with TRIzol (Thermo Fisher Scientific, Inc.) according to the manufacturer's protocols. Then, cDNA was synthesized with TaqMan MicroRNA Reverse Transcription Kit (Applied Biosystems; Thermo Fisher Scientific, Inc.). Subsequently, qPCR was performed using a TaqMan MicroRNA Assay kit (Applied Biosystems; Thermo Fisher Scientific, Inc.) with an ABI 7300 qPCR system (Applied Biosystems; Thermo Fisher Scientific, Inc.). The thermocycling conditions used for qPCR were as follows: Denaturation at $95^{\circ} \mathrm{C}$ for $10 \mathrm{~min}$; followed by 40 cycles of denaturation at $95^{\circ} \mathrm{C}$ for $15 \mathrm{sec}$ and elongation at $60^{\circ} \mathrm{C}$ for $1 \mathrm{~min}$. The relative expression levels of SATB2 and miRs were calculated and normalized to that of endogenous $\beta$-actin or U6, respectively. The relative fold change in expression was calculated by the $2^{-\Delta \Delta \mathrm{Cq}}$ method (21). The primer sequences used were as follows: U6 forward, 5'-AACGAGACGACGACAGAC-3' and reverse, 5'-GCAAATTCGTGAAGCGTTCCATA-3'; $\beta$-actin forward, 5'-CGGCGCCCTATAAAACCCA-3' and reverse, 5'-GAGGCGTACAGGGATAGCAC-3'; miR-3666 forward, 5'-AACGAGACGACGACAGAC-3' and reverse, 5'-CAGTGC AAGTGTAGATGCCGA-3'; SATB2 forward, 5'-GCCTGA TGACTCAACGCAAC-3' and reverse, 5'-ACACCAAGAGCC GAGAAGAC-3'. All assays were performed in triplicate.

Western blot analysis. CRC cells were lysed using radioimmunoprecipitation buffer (Thermo Fisher Scientific, Inc.) and total protein lysates were obtained. Protein concentration was determined using a bicinchoninic acid assay. Total protein $(20 \mu \mathrm{g})$ was separated using $4-20 \%$ SDS-PAGE gels and then transferred to polyvinylidene fluoride membranes at $4^{\circ} \mathrm{C}$. Membranes were subsequently blocked at $25^{\circ} \mathrm{C}$ for $1 \mathrm{~h}$ with $5 \%$ non-fat milk/Tris-buffered saline containing $0.1 \%$ Tween-20, and then incubated at $4^{\circ} \mathrm{C}$ overnight with primary antibodies against the following proteins: SATB2 (1:2,000; cat. no. 39229; Cell Signaling Technology, Inc., Danvers, MA, USA) and GAPDH (1:2,000; cat. no. 5174; Cell Signaling Technology, Inc.). Following this, membranes were incubated with goat anti-rabbit horseradish peroxidase (HRP)-conjugated secondary antibodies (1:2,000; cat. no. 7074; Cell Signaling Technology, Inc.) for $2 \mathrm{~h}$ at $25^{\circ} \mathrm{C}$. Protein signals on membranes were detected using enhanced chemiluminescence reagents (GE Healthcare, Chicago, IL, USA). 
Immunohistochemistry. CRC tissues were fixed in $10 \%$ formaldehyde at $4^{\circ} \mathrm{C}$ for $12 \mathrm{~h}$ and then embedded in paraffin. Following this, tissues were cut into $6 \mu \mathrm{m}$ sections, placed on silanized glass slides, deparaffinized in xylene and then rehydrated using ethanol gradients. The sections were then pretreated with antigen target retrieval solution [10 mmol/1 citric acid monohydrate adjusted with $2 \mathrm{nM}$ sodium hydroxide to (pH 6.0)] at $90^{\circ} \mathrm{C}$ for $40 \mathrm{~min}$ in citrate buffer. Endogenous peroxidase activity was blocked via incubation with methanol with $0.3 \%$ hydrogen peroxide for $30 \mathrm{~min}$ at room temperature. Sections were then incubated with anti-SATB2 (SATB2; 1:500; cat. no. 39229; Cell Signaling Technology, Inc.) at $4^{\circ} \mathrm{C}$ for $12 \mathrm{~h}$. Following this, tumor tissues were incubated with goat anti-rabbit HRP-conjugated secondary antibodies $(1: 2,000$; cat. no. 7074; Cell Signaling Technology, Inc.) for $1 \mathrm{~h}$ at $37^{\circ} \mathrm{C}$. Proteins were then visualized using the DAKO EnVision system (Dako; Agilent Technologies GmbH, Waldbronn, Germany) in accordance with the manufacturer's instructions and images were captured using a light microscope (magnification, x100).

Luciferase reporter assay. The 3'-UTR region of SATB2 containing the binding site of miR-3666 was constructed into the pmirGLO vector (Promega Corporation, Madison, WI, USA). In order to perform luciferase reporter assays, $2 \times 10^{4}$ HCT116 and SW480 cells per well were plated in 96-well plates and then incubated at $37^{\circ} \mathrm{C}$ for $24 \mathrm{~h}$. Subsequently, the cells were transfected with miR-3666 mimics and pmirGLO-SATB2-3'UTR or pmirGLO-3'UTR-MUT plasmids using Lipofectamine ${ }^{\circledR} 2000$ (Invitrogen; Thermo Fisher Scientific, Inc.) according to the manufacturer's protocol. A total of $24 \mathrm{~h}$ post-transfection, luciferase activity was measured using the Dual-Luciferase Reporter Assay System (Promega Corporation). Luciferase activity was normalized to Renilla luciferase activity.

Statistical analysis. Data were expressed as the mean \pm standard deviation of 3 independent experiments. Statistical analysis was performed using SPSS software version 20.0 (IBM Corp., Armonk, NY, USA). The statistical significance of the differences between groups was assessed using a Student's t-test or one-way analysis of variance followed by a Tukey's post-hoc test for multiple comparisons. The association between miR-3666 levels and the clinical features of patients with CRC was investigated via Chi-square test. Pearson's correlation analysis was used to determine correlations between miR-3666 and SATB1 in expression levels. Survival curves were calculated using the Kaplan-Meier method and were analyzed using with a log-rank test. $\mathrm{P}<0.05$ was considered to indicate a statistically significant difference.

\section{Results}

miR-3666 is downregulated in CRC tissues. The expression levels of miR-3666 in $53 \mathrm{CRC}$ tissues and 53 adjacent normal tissues were analyzed by RT-qPCR. The results revealed that the mean expression levels of miR-3666 were significantly lower in CRC tissues compared with in adjacent normal tissues (Fig. 1A). In addition, compared with in the normal control cell line NCM470, the expression levels of miR-3666 were significantly downregulated in CRC cell lines (HT29,
HCT116, SW480 and SW620 cells; Fig. 1B). Furthermore, the association between miR-3666 expression and the clinical characteristics of patients with CRC was determined. The results of the present study revealed that miR-3666 expression levels were negatively correlated with $\mathrm{CRC}$ tumor, node and metastasis (TNM) stage, tumor size, and metastasis (Table I); however, no association was observed between miR-3666 expression and other clinicopathological characteristics, including age and gender. To analyze the association between miR-3666 expression and prognosis, Kaplan-Meier survival analysis was performed. The results revealed that more patients with higher expression levels of miR-3666 exhibited longer survival times $(\mathrm{P}=0.037$; Fig. $1 \mathrm{C})$. These results indicated that miR-3666 was aberrantly expressed in CRC tissues and may participate in the progression of CRC.

miR-3666 suppresses the proliferation, migration and invasion of CRC cells. To investigate the function of miR-3666 in CRC, miR-3666 overexpressed or downregulated in HCT116 and SW480 cells by transfection with miR-3666 mimics, inhibitors or miR-NC, respectively. RT-qPCR analysis indicated that, compared with in the control group, miR-3666 was significantly overexpressed and notably downregulated following the transfection of HCT116 and SW480 cells with miR-3666 mimics and inhibitors, respectively (Fig. 2A). Then, a CCK-8 assay was conducted to determine the potential of cellular proliferation. The results demonstrated that, compared with in the control group, overexpression of miR-3666 significantly inhibited cell proliferation and vice versa (Fig. 2B). Additionally, Transwell assays were used to evaluate cell migration and invasion. The results revealed that overexpression of miR-3666 significantly suppressed the number of migrated and invaded cells, whereas inhibition of miR-3666 significantly promoted cell migration and invasion compared with in the control group (Fig. 2C and D). These data indicated that miR-3666 suppressed the proliferation, migration and invasion of CRC cells.

SATB2 is a target gene of miR-3666. To further investigate the downstream mechanism, the target gene of miR-3666 in CRC cells was determined. Analysis with TargetScan 7.0 revealed that numerous genes were potential targets of miR-3666. Among these targets, SATB2 ranked first and has been reported to promote the development of CRC (22); the potential binding site of miR-3666 in the 3'-UTR of SATB2 was presented in Fig. 3A. Therefore, SATB2 was selected for investigation in the present study. Using luciferase reporter assays, it was demonstrated that overexpression of miR-3666 significantly inhibited the luciferase activity of HCT116 and SW480 cells transfected with the wild type 3'-UTR, whereas knockdown of miR-3666 significantly upregulated luciferase activity compared with in the control (Fig. 3B). Furthermore, the present study revealed that overexpression of miR-3666 significantly enhanced the mRNA and notably increased the protein expression levels of SATB2 in HCT116 and SW480 cells compared with in the control (Fig. 3C and D). In addition, RT-qPCR analysis indicated that the expression of miR-3666 was negatively correlated with that of SATB2 in CRC tissues (Fig. 3E). Then, the expression of SATB2 in CRC tissues was analyzed by RT-qPCR, which demonstrated 
Table I. Association between miR-3666 expression and clinicopathological features of patients with CRC.

\begin{tabular}{|c|c|c|c|c|}
\hline Features & Cases & Low expression & High expression & P-value \\
\hline Sex & & & & 0.414 \\
\hline Male & 28 & 12 & 16 & \\
\hline Female & 25 & 14 & 11 & \\
\hline Age (years) & & & & 0.275 \\
\hline$<60$ & 30 & 16 & 15 & \\
\hline$\geq 60$ & 23 & 10 & 12 & \\
\hline Tumor size $(\mathrm{cm})$ & & & & $0.028^{\mathrm{a}}$ \\
\hline$<5$ & 25 & 8 & 17 & \\
\hline$\geq 5$ & 28 & 18 & 10 & \\
\hline Lymph node metastasis & & & & $0.028^{\mathrm{a}}$ \\
\hline Absent & 29 & 10 & 19 & \\
\hline Present & 24 & 16 & 8 & \\
\hline TNM stage & & & & $0.006^{\mathrm{a}}$ \\
\hline I-II & 25 & 7 & 18 & \\
\hline III-IV & 28 & 19 & 9 & \\
\hline
\end{tabular}

The association between miR-3666 levels and the clinical features of patients with CRC was analyzed using the Chi-square test. ${ }^{\mathrm{a} P}<0.05$ was considered to represent a statistically significant difference. CRC, colorectal cancer; miR, microRNA; TNM, tumor, node and metastasis.
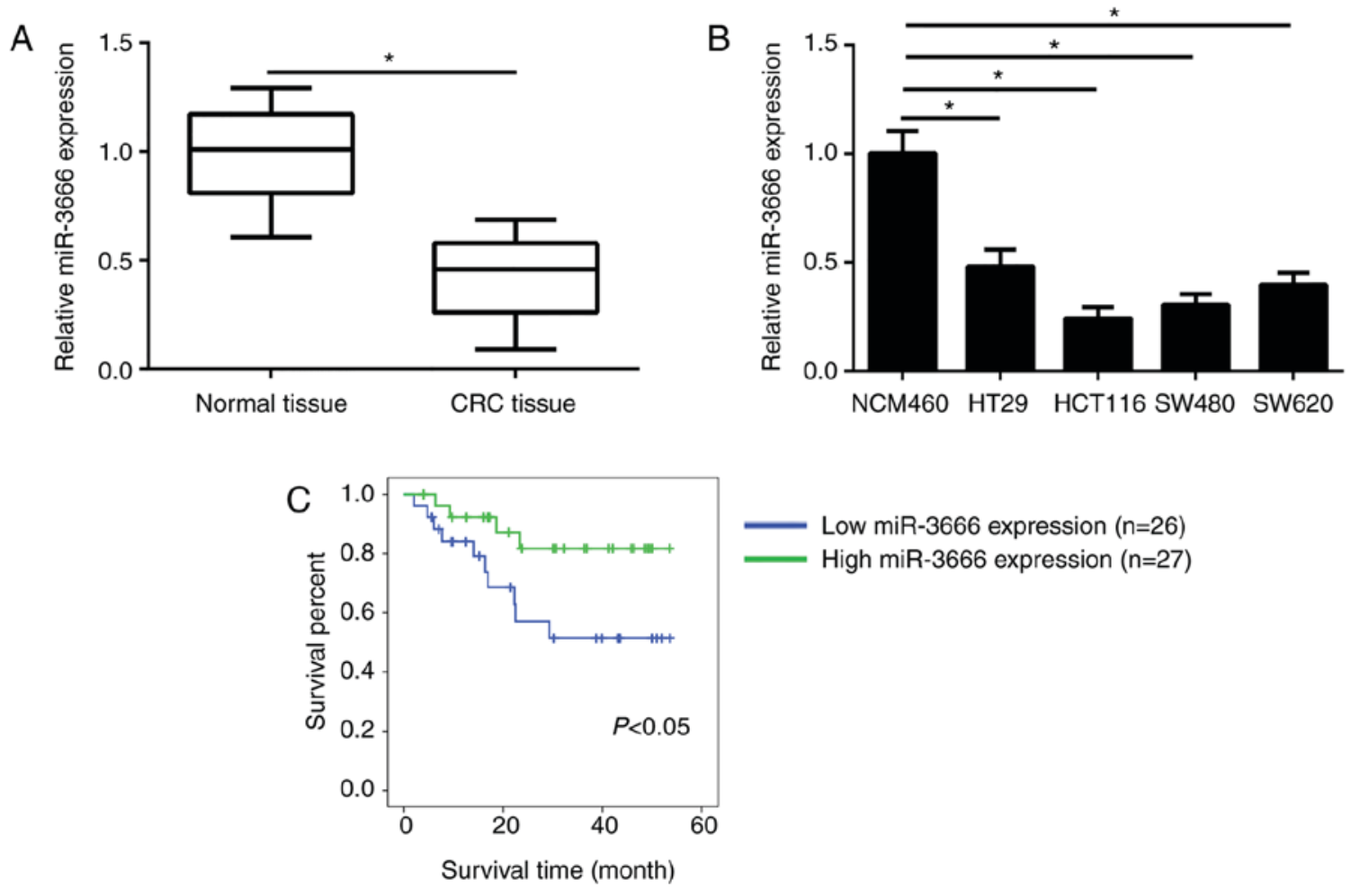

Figure 1. miR-3666 is downregulated in CRC tissues. (A) Relative expression of miR-3666 was measured by RT-qPCR in CRC tissues ( $\mathrm{n}=53$ ) and adjacent normal tissues ( $\mathrm{n}=53$ ). (B) Relative expression of miR-3666 in CRC cell lines was determined by RT-qPCR. (C) Kaplan-Meier survival analysis revealed the association between miR-3666 expression in CRC tissues patient survival. " $\mathrm{P}<0.05$ vs. NC group or NCM460 cells. CRC, colorectal cancer; miR, microRNA; $\mathrm{NC}$, negative control; RT-qPCR, reverse transcription-quantitative polymerase chain reaction.

that the expression of SATB2 was significantly upregulated in CRC tissues compared with in adjacent normal tissues (Fig. 3F). Furthermore, immunohistochemical and western blot analyses indicated that SATB2 was overexpressed in
CRC tissues compared with in matched normal tissues (Fig. 3G and H). Collectively, the results of the study indicated that miR-3666 targeted SATB2, which was upregulated in CRC tissues. 


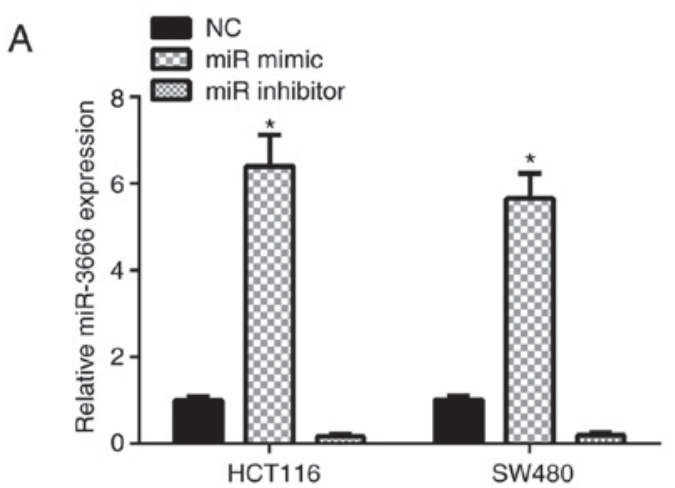

B
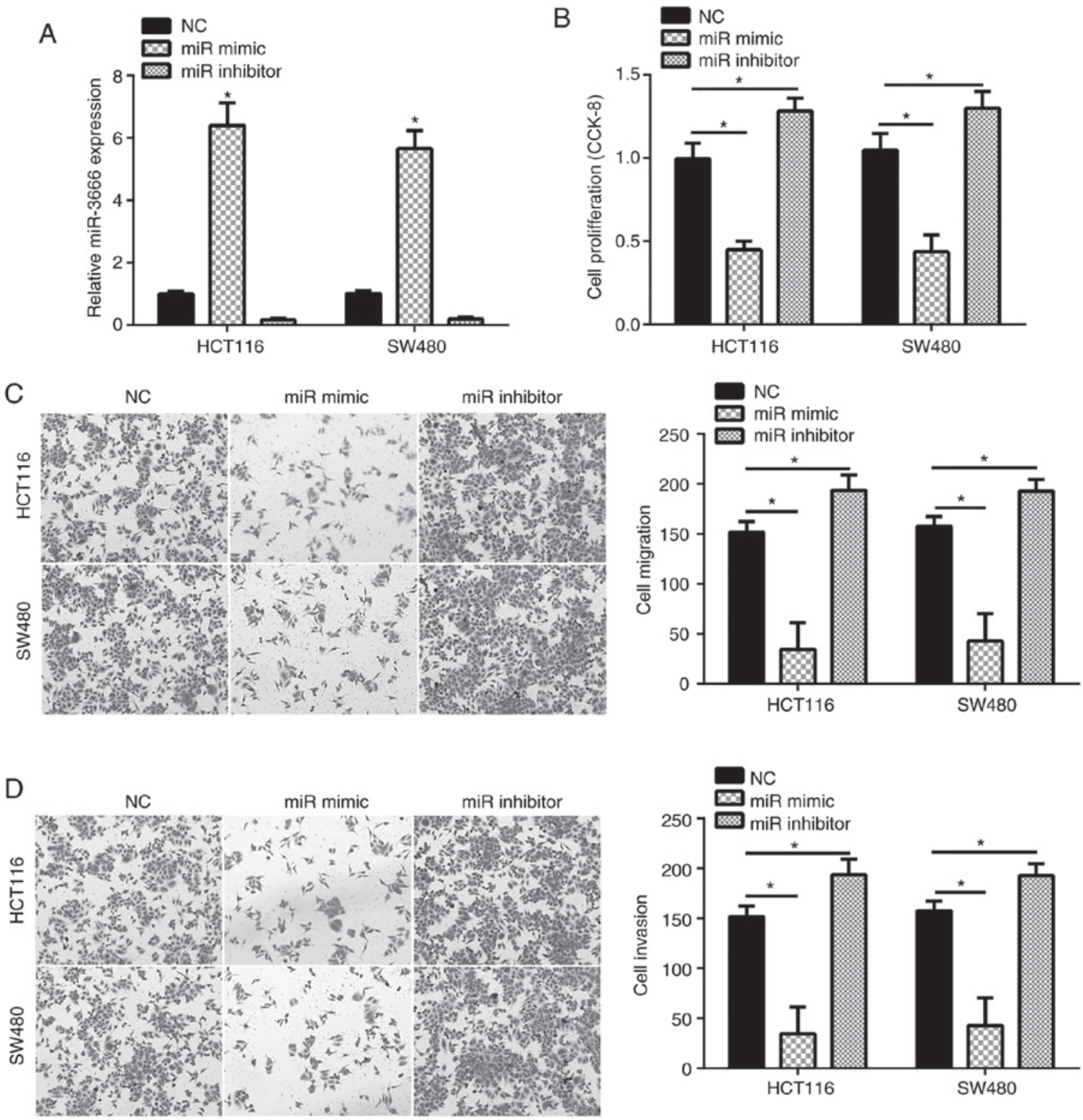

Figure 2. miR-3666 suppresses the proliferation, migration and invasion of colorectal cancer cells. (A) Relative expression levels of miR-3666 were measured by reverse transcription-quantitative polymerase chain reaction in HCT116 and SW480 cells transduced with miR-3666 mimics, inhibitors or NC. (B) Cell proliferation was analyzed via a CCK-8 assay. (C and D) Transwell assay was used to examine cell migration and invasion (magnification, $\mathrm{x} 100)$. "P $<0.05$ vs. NC group. CCK-8, Cell Counting Kit-8; miR, microRNA; NC, negative control.

Restoration of SATB2 expression abolishes the antitumor effects of miR-3666. To determine whether SATB2 is required for miR-3666-mediated effects on CRC cells, the protein expression levels of SATB2 were restored in HCT116 and SW480 cells transfected with miR-3666. Western blotting indicated that the expression of SATB2 protein was notably increased in HCT116 and SW480 cells expressing the SATB2 overexpression vector compared with in cells transfected with miR-3666 mimics (Fig. 4A). Then, CCK-8 and Transwell assays were conducted, which revealed that cell proliferation, migration and invasion was significantly inhibited in cells overexpressing miR-3666 compared with in the control; however, restoration of SATB2 significantly reversed these effects of miR-3666 (Fig. 4B-D). In conclusion, the data suggested that miR-3666 suppressed CRC cell proliferation, migration and invasion by targeting SATB2.

\section{Discussion}

CRC is one of the most malignant types of cancer and one of the leading cause of cancer-associated mortality worldwide (23). Improvements in the treatment of CRC have been reported; however, the prognosis of patients with CRC remains poor (1). Numerous patients with CRC are diagnosed at advanced stages when tumor metastasis occurs (2). Therefore, in order to identify biomarkers and therapeutic targets for the diagnosis, prognosis and treatment of CRC, complete understanding of the underlying molecular mechanisms of CRC progression is required (24). Increasing evidence has indicated that miRNAs regulate cell proliferation, survival and metastasis (25). Numerous miRNAs have been reported as promising biomarkers for CRC (26). Thus, miRNAs may be considered as important regulators in tumor progression. 


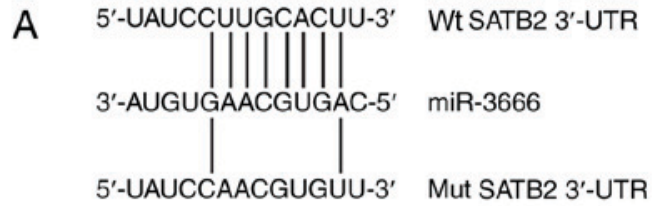

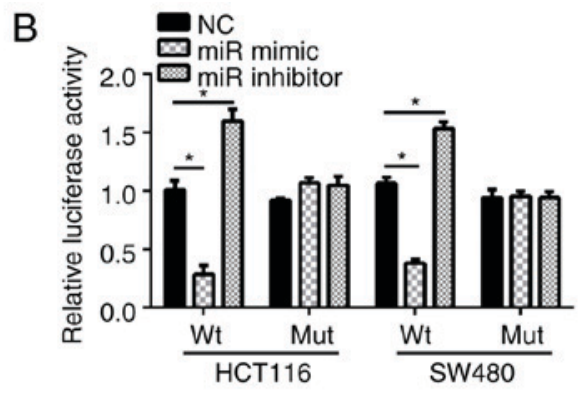

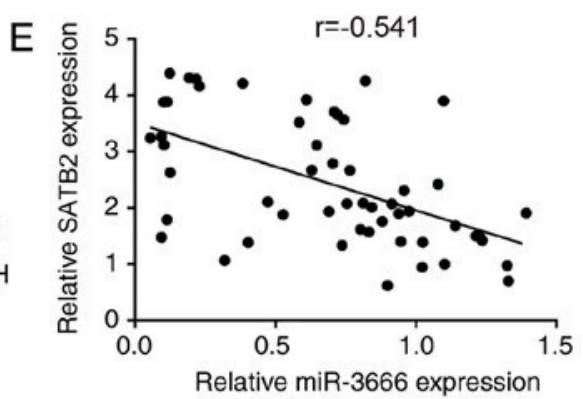

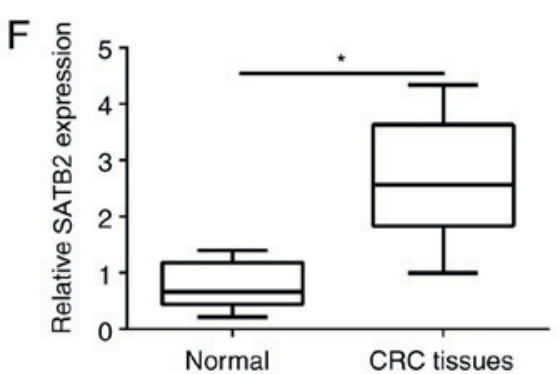
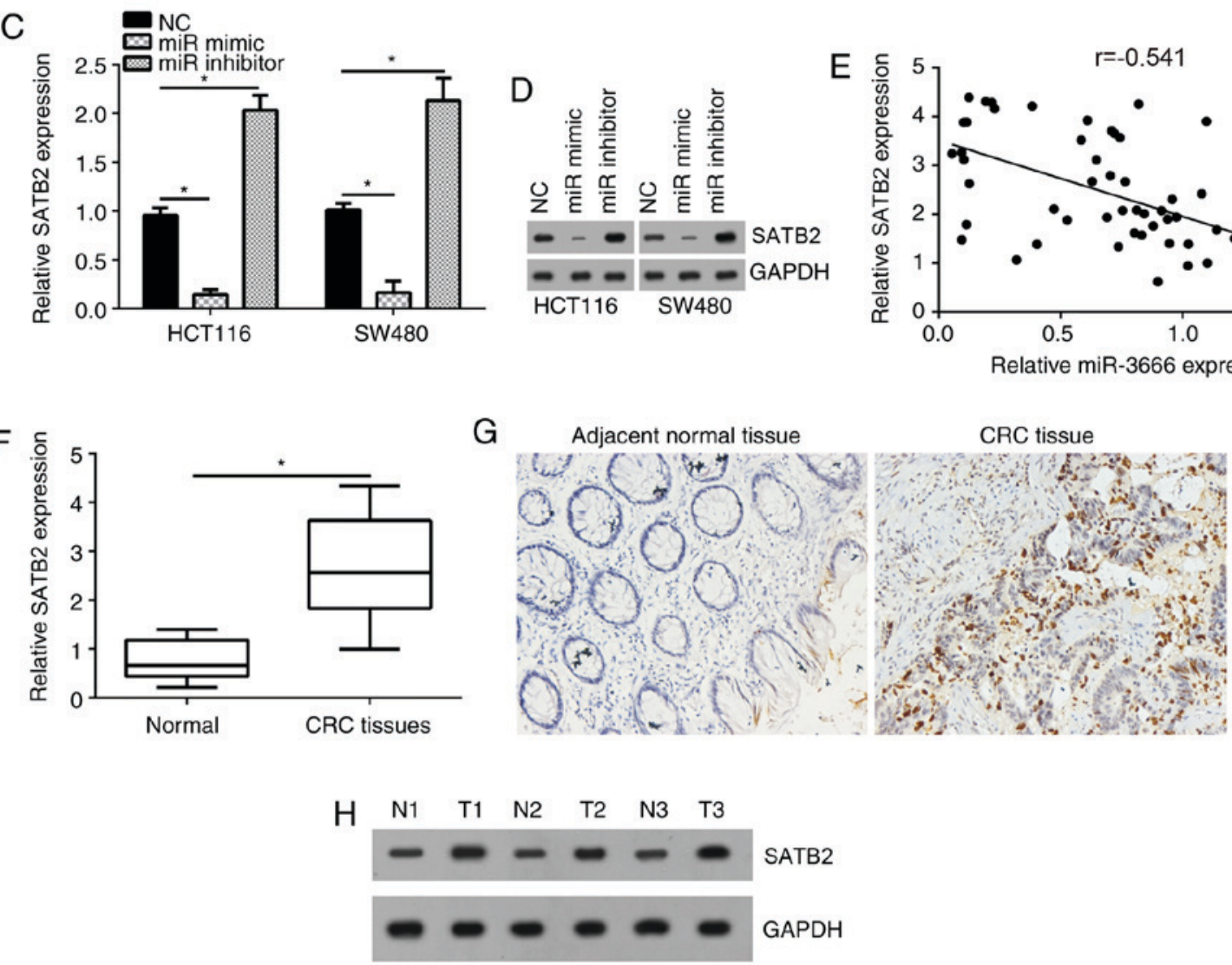

Figure 3. SATB2 is a target gene of miR-3666. (A) A diagram for the potential binding site of miR-3666 in the 3'-UTR of SATB2 mRNA. (B) A SATB2 3'-UTR fragment containing the Wt or Mut miR-3666 binding site was cloned into the pmirGLO reporter gene vector. Cells were cotransfected with the reporter gene construct vector and miR-3666 mimics or inhibitor for $48 \mathrm{~h}$. (C and D) HCT116 and SW480 cells were transfected with miR-3666 mimics or inhibitor for $48 \mathrm{~h}$. Relative mRNA and protein levels of SATB2 were detected by RT-qPCR and western blotting. (E) Correlation between SATB2 mRNA and miR-3666 expression was assessed by Pearson's correlation analysis. (F) Relative expression of SATB2 was measured by RT-qPCR in CRC tissues and adjacent normal tissues. (G) SATB2 expression was determined by immunohistochemical analysis in paired CRC tissues and normal tissues (magnification, $\mathrm{x} 100)$. (H) Western blot analysis of SATB2 protein expression in 3 pairs of CRC tissues and adjacent normal tissues. "P $<0.05$ vs. NC group or normal tissues. miR, microRNA; Mut, mutant; N, normal tissue; NC, negative control; RT-qPCR, reverse transcription-quantitative polymerase chain reaction; SATB2, special AT-rich sequence binding protein 2; T, tumor tissue; UTR, untranslated region; Wt, wild type.

For example, miRNA-520c was demonstrated to promote gastric cancer growth and metastasis via targeting interferon regulatory factor 2 (27). In addition, miR-451 was observed to enhance pancreatic cancer growth via inhibition of calcium-binding protein 39 (28). miR-381 suppressed gastric cancer cell migration and invasion by inhibiting transmembrane member 16A (29).

The function of miR-3666 has been recently studied $(17,18)$. Shi et al (17) reported that miR-3666 inhibited lung cancer cell proliferation by targeting sirtuin 7 . Wang et al (18) revealed that miR-3666 upregulation suppressed the progression of thyroid carcinoma. Additionally, Li et al (19) reported that miR-3666 overexpression repressed cervical cancer growth and metastasis. Collectively, these data indicated that miR-3666 serves as a tumor suppressor in certain types of cancer; however, its role and clinical significance in CRC remain unknown. In the present study, it was reported that miR-3666 was downregulated in CRC tissues compared with in adjacent normal tissues. In addition, miR-3666 expression was associated with tumor size, TNM staging and metastasis in CRC, and the downregulation of miR-3666 was associated with a poor prognosis of CRC. Functional experiments demonstrated that miR-3666 suppressed the proliferation, migration and invasion of CRC cells. The results of the present study indicated that miR-3666 serves as a tumor suppressor in CRC and as a potential biomarker for the prognosis of CRC. 
A
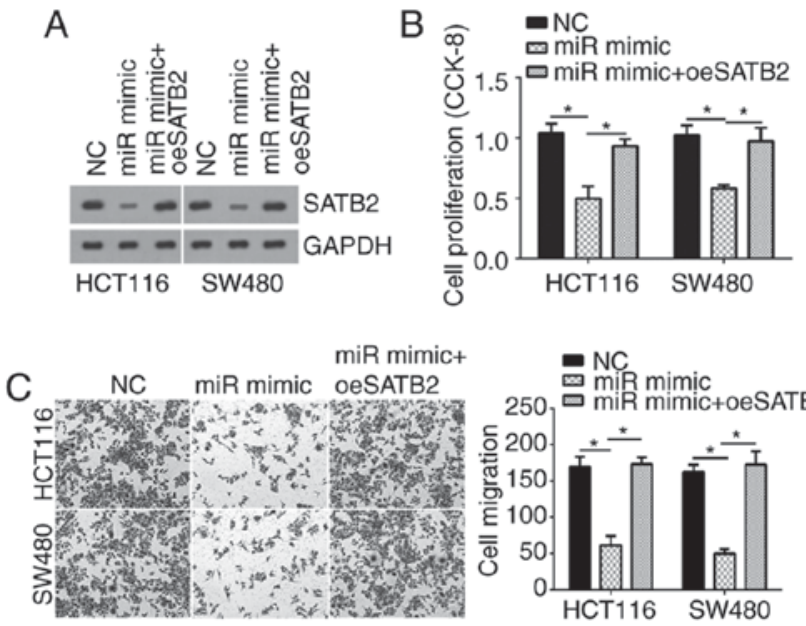

miR mimict
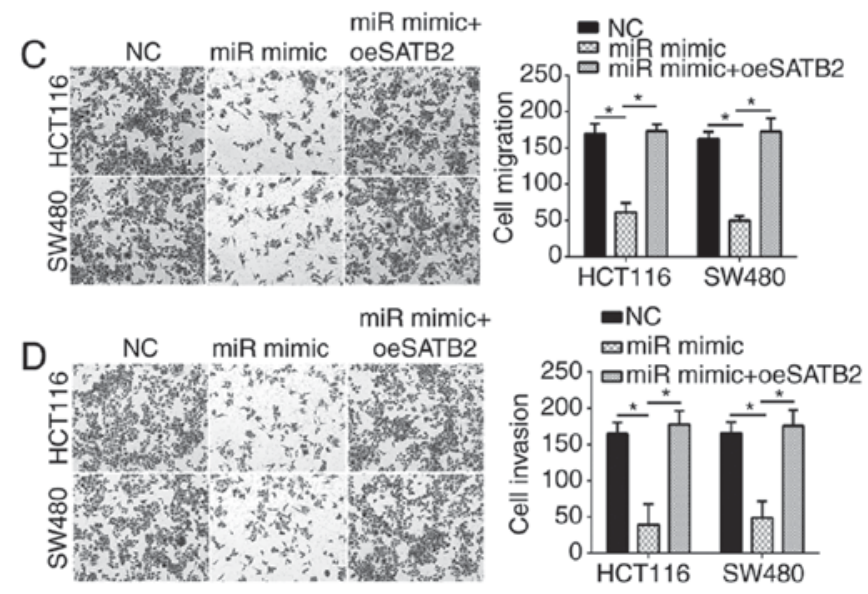

Figure 4. Restoration of SATB2 expression abolishes the antitumor effects of miR-3666. (A) Protein expression of SATB2 was detected by western blotting in SW480 and HCT116 cells cotransfected with miR-3666 mimics, miR-3666 mimics+pcDNA3.1-SATB2 or negative controls. (B) Cell proliferation was detected via a CCK-8 assay. (C and D) Cell migration and invasion were detected via Transwell assays (magnification, $\mathrm{x} 100$ ). ${ }^{*} \mathrm{P}<0.05$. CCK- 8 , Cell Counting Kit-8; miR, microRNA; NC, negative control; oe, overexpression; SATB2, special AT-rich sequence binding protein 2 .

To investigate the molecular mechanism underlying miR-3666-suppressed CRC cell proliferation and metastasis, the target gene of miR-3666 was determined; SATB2 was predicted as a direct target gene of miR-3666 in CRC cells in the present study. SATB2 is a transcription factor that has been associated with tumorigenesis (30). For instance, Wang et al (20) reported that SATB2 promoted lung cancer metastasis. Luo et al (31) demonstrated that SATB2 induced the progression of triple negative breast cancer. A recent study also indicated that SATB2 promoted the progression of colorectal cancer via the Wnt pathway (30). These studies demonstrated the role of SATB2 as an oncogene in a variety of cancers; however, the regulation of SATB2 expression in $\mathrm{CRC}$ requires further investigation. In the present study, using a luciferase reporter assay, miR-3666 was proposed to directly target SATB2 in CRC cells. Additionally, the findings of the present study suggested that miR-3666 significantly inhibited the expression of SATB2; miR-3666 expression was negatively correlated with that of SATB2 in CRC tissues. In addition, SATB2 expression was significantly upregulated in CRC tissues and cell lines. Furthermore, via a series of functional experiments, the present study demonstrated that restoration of SATB2 expression rescued the effects of miR-3666 on CRC cell proliferation, migration and invasion.

In summary, miR-3666 was reported to be downregulated in CRC tissues and overexpression of miR-3666 impaired the proliferation, migration and invasion of CRC cells by targeting SATB2 in the present study. Collectively, these results may provide novel insight to improve understanding of the mechanism underlying miR-3666 and SATB2 in the regulation of CRC progression, and may aid the identification of potential therapeutic targets for the clinical treatment of CRC.

\section{Acknowledgements}

Not applicable.

\section{Funding}

No funding was received.

\section{Availability of data and materials}

All data generated or analyzed during this study are included in this published article.

\section{Authors' contributions}

DY and HZ made substantial contributions to the design of the present study, analyzed and interpreted the results, and wrote the manuscript. RL, JX and WL performed some experiments. All authors read and approved the final manuscript.

\section{Ethics approval and consent to participate}

For the use of human samples, the protocol employed in the present study was approved by the Institutional Ethics Committee of Wenzhou Central Hospital (Wenzhou, China) and all enrolled patients provided written informed consent.

\section{Patient consent for publication}

Not applicable.

\section{Competing interests}

The authors declare that they have no competing interests.

\section{References}

1. Vermeer NC, Snijders HS, Holman FA, Liefers GJ, Bastiaannet E, van de Velde $\mathrm{CJ}$ and Peeters KC: Colorectal cancer screening: Systematic review of screen-related morbidity and mortality. Cancer Treat Rev 54: 87-98, 2017.

2. Ballester V, Rashtak S and Boardman L: Clinical and molecular features of young-onset colorectal cancer. World J Gastroenterol 22: 1736-1744, 2016.

3. Zafar SY, McNeil RB, Thomas CM, Lathan CS, Ayanian JZ and Provenzale D: Population-based assessment of cancer survivors' financial burden and quality of life: A prospective cohort study. J Oncol Pract 11: 145-150, 2015.

4. Pan Y, Tong JHM, Lung RWM, Kang W, Kwan JSH, Chak WP, Tin KY, Chung LY, Wu F, Ng SSM, et al: RASAL2 promotes tumor progression through LATS2/YAP1 axis of hippo signaling pathway in colorectal cancer. Mol Cancer 17: 102, 2018.

5. Yu W, Wang Y and Guo P: Notch signaling pathway dampens tumor-infiltrating $\mathrm{CD} 8^{+} \mathrm{T}$ cells activity in patients with colorectal carcinoma. Biomed Pharmacother 97: 535-542, 2018.

6. Sakai E, Nakayama M, Oshima H, Kouyama Y, Niida A, Fujii S, Ochiai A, Nakayama KI, Mimori K, Suzuki Y, et al: Combined mutation of Apc, Kras and Tgfbr2 effectively drives metastasis of intestinal cancer. Cancer Res 78: 1334-1346, 2018. 
7. Esquela-Kerscher A and Slack FJ: Oncomirs-microRNAs with a role in cancer. Nat Rev Cancer 6: 259-269, 2006.

8. Kloosterman WP and Plasterk RH: The diverse functions of microRNAs in animal development and disease. Dev Cell 11: 441-450, 2006.

9. Mei LL, Qiu YT, Wang WJ, Bai J and Shi ZZ: Overexpression of microRNA-1470 promotes proliferation and migration, and inhibits senescence of esophageal squamous carcinoma cells. Oncol Lett 14: 7753-7758, 2017.

10. Wang $G$, Fang $X$, Han $M$, Wang $X$ and Huang Q: MicroRNA-493-5p promotes apoptosis and suppresses proliferation and invasion in liver cancer cells by targeting VAMP2. Int J Mol Med 41: 1740-1748, 2018.

11. Slaby O, Svoboda M, Michalek J and Vyzula R: MicroRNAs in colorectal cancer: Translation of molecular biology into clinical application. Mol Cancer 8: 102, 2009.

12. Koo KH and Kwon H: MicroRNA miR-4779 suppresses tumor growth by inducing apoptosis and cell cycle arrest through direct targeting of PAK2 and CCND3. Cell Death Dis 9: 77, 2018

13. Islam F, Gopalan V, Vider J, Lu CT and Lam AK: miR-142-5p act as an oncogenic microRNA in colorectal cancer: Clinicopathological and functional insights. Exp Mol Pathol 104 98-107, 2018.

14. He S, Wang G, Ni J, Zhuang J, Zhuang S, Wang G, Ye Y and Xia W: MicroRNA-511 inhibits cellular proliferation and invasion in colorectal cancer by directly targeting hepatoma-derived growth factor. Oncol Res: Jan 10, 2018 (Epub ahead of print)

15. Alipour A, Mojdehfarahbakhsh A, Tavakolian A Morshedzadeh T, Asadi M, Mehdizadeh A and Nami M: Neural communication through theta-gamma cross-frequency coupling in a bistable motion perception task. J Integr Neurosci 15: 539-551, 2016

16. Yuan Z, Baker K, Redman MW, Wang L, Adams SV, Yu M, Dickinson B, Makar K, Ulrich N, Böhm J, et al: Dynamic plasma microRNAs are biomarkers for prognosis and early detection of recurrence in colorectal cancer. Br J Cancer 117: 1202-1210, 2017.

17. Shi H, Ji Y,Zhang D, Liu Y and Fang P: MicroRNA-3666-induced suppression of SIRT7 inhibits the growth of non-small cell lung cancer cells. Oncol Rep 36: 3051-3057, 2016.

18. Wang G, Cai C and Chen L: MicroRNA-3666 regulates thyroid carcinoma cell proliferation via MET. Cell Physiol Biochem 38 1030-1039, 2016

19. Li L, Han LY, Yu M, Zhou Q, Xu JC and Li P: Pituitary tumor-transforming gene 1 enhances metastases of cervical cancer cells through miR-3666-regulated ZEB1. Tumour Biol: Sep 17, 2015 (Epub ahead of print).
20. Wang J, Lu Y, Ding H, Gu T, Gong C, Sun J, Zhang Z, Zhao Y and Ma C: The miR-875-5p inhibits SATB2 to promote the invasion of lung cancer cells. Gene 644: 13-19, 2018.

21. Livak KJ and Schmittgen TD: Analysis of relative gene expression data using real-time quantitative PCR and the 2(-Delta Delta C(T)) method. Methods 25: 402-408, 2001.

22. Sun X, Liu S, Chen P, Fu D, Hou Y, Hu J, Liu Z, Jiang Y, Cao X, Cheng $\mathrm{C}$, et al: $\mathrm{miR}-449 \mathrm{a}$ inhibits colorectal cancer progression by targeting SATB2. Oncotarget 8: 100975-100988, 2017.

23. Qu JJ, Qu XY and Zhou DZ: miR4262 inhibits colon cancer cell proliferation via targeting of GALNT4. Mol Med Rep 16: 3731-3736, 2017

24. Dowling CM, Hayes SL, Phelan JJ, Cathcart MC, Finn SP, Mehigan B, McCormick P, Coffey JC, O'sullivan J and Kiely PA: Expression of protein kinase $\mathrm{C}$ gamma promotes cell migration in colon cancer. Oncotarget 8: 72096-72107, 2017.

25. Kok MG, Mandolini C, Moerland PD, de Ronde MW, Sondermeijer BM, Halliani A, Nieuwland R, Cipollone F, Creemers EE, Meijers JC and Pinto-Sietsma SJ: Low miR-19b-1-5p expression in isolated platelets after aspirin use is related to aspirin insensitivity. Int J Cardiol 203: 262-263, 2016.

26. Hu WL and Zhou XH: Identification of prognostic signature in cancer based on DNA methylation interaction network. BMC Med Genomics 10 (Suppl 4): S63, 2017.

27. Li YR, Wen LQ, Wang Y, Zhou TC, Ma N, Hou ZH and Jiang ZP: MicroRNA-520c enhances cell proliferation, migration, and invasion by suppressing IRF2 in gastric cancer. Febs Open Bio 6: $1257-1266,2016$.

28. Guo R, Gu J, Zhang Z, Wang Y and Gu C: miR-451 promotes cell proliferation and metastasis in pancreatic cancer through targeting CAB39. Biomed Res Int 2017: 2381482, 2017.

29. Cao Q, Liu F, Ji K, Liu N, He Y, Zhang W and Wang L: MicroRNA-381 inhibits the metastasis of gastric cancer by targeting TMEM16A expression. J Exp Clin Cancer Res 36: 29 , 2017.

30. Yu W, Ma Y, Shankar S and Srivastava RK: SATB2/ $\beta$-catenin/TCF-LEF pathway induces cellular transformation by generating cancer stem cells in colorectal cancer. Sci Rep 7: 10939, 2017.

31. Luo LJ, Yang F, Ding JJ, Yan DL, Wang DD, Yang SJ, Ding L, Li J, Chen D, Ma R, et al: miR-31 inhibits migration and invasion by targeting SATB2 in triple negative breast cancer. Gene 594: 47-58, 2016.

This work is licensed under a Creative Commons Attribution-NonCommercial-NoDerivatives 4.0 International (CC BY-NC-ND 4.0) License. 